\title{
En quoi différentes approches de l'activité collective des relations de services interrogent la pluralité des modèles de l'activité en ergonomie?
}

How do different approaches to collective activity in service relations call into question the plurality of ergonomic activity models?

\section{Sandrine Caroly et Annie Weill-Fassina}

\section{OpenEdition}

Journals

Édition électronique

URL : http://journals.openedition.org/activites/1414

DOI : 10.4000/activites. 1414

ISSN : $1765-2723$

Éditeur

ARPACT - Association Recherches et Pratiques sur les ACTivités

\section{Référence électronique}

Sandrine Caroly et Annie Weill-Fassina, « En quoi différentes approches de l'activité collective des relations de services interrogent la pluralité des modèles de l'activité en ergonomie ? ", Activités [En ligne], 4-1 | avril 2007, mis en ligne le 15 avril 2007, consulté le 19 avril 2019. URL : http:// journals.openedition.org/activites/1414; DOI : 10.4000/activites.1414

\section{(c) $($ ) (9)}

Activités est mis à disposition selon les termes de la licence Creative Commons Attribution - Pas d'Utilisation Commerciale - Pas de Modification 4.0 International. 


\title{
En quoi différentes approches de l'activité collective des relations de services interrogent la pluralité des modèles de l'activité en ergonomie?
}

\author{
Sandrine Caroly \\ Centre de Recherches en Innovations SocioTechniques et Organisations Industrielles, \\ Université Grenoble 2, UPMF, BP 47, 39040 Grenoble cedex 9, France. \\ Centre de Recherches et d'Etudes sur l'Age des Populations au Travail, Paris \\ Sandrine.Caroly@upmf-grenoble.fr
}

\begin{abstract}
Annie Weill-Fassina
Laboratoire d'Ergonomie Physiologique et Cognitive, Ecole pratique des Hautes Etudes, 41 rue Gay Lussac, 75005 Paris, France
\end{abstract}

\begin{abstract}
How do different approaches to collective activity in service relations call into question the plurality of ergonomic activity models?

The aim of this paper is to show that the various characteristics of service relationships in public utilities and demands about them require careful thought concerning what we call "activity". Following task analysis (nurses, office clerks, policemen, funeral directors, crèche, nurses, educators...) don't do the same work: their aim, the types of interactions between "customers" and workers, work conditions and organization, possibilities of service relations. So, the problem is to precise what aspects of workers' activities, we have to take into account so that activity analysis can be oriented to a more adequate understanding of the job and relevant ergonomic interventions. In order to take action about health and efficiency, they can often require passing beyond a behavioral analysis which describes effective task or its observable components, according to work conditions. We need to have a better understanding of the internal activity component that is a better understanding of physiological, affective and cognitive mechanisms that direct and regulate this behaviour according to the operators' motives, goals and possibilities in interaction with the environmental conditions. To render an account of reality, this kind of pragmatic systemic analysis of activity has to be supported by different "activity theories" according to the research purpose and conditions.
\end{abstract}

KEYWORDS

Activity Theory, Service Activities, Regulations, Collaborative Work, Organization.

\section{1.- Une multiplicité de points de vue dans l'analyse des relations de service}

Le but de ce texte est de montrer et de caractériser, sans vouloir être exhaustif, la diversité des modalités d'activités individuelles et collectives que recouvre la notion d'« activité de travail » dans les relations de service du secteur public. 
Les « analyses du travail » que nous avons pu faire dans différents métiers, ont montré l'extrême diversité des buts, des tâches, des types d'interactions avec le « client», des conditions du travail et de son organisation. Ces recherches interrogent les modalités d'analyse et les théories de l'activité.

\section{1.-Diversification des « relations de service » dans les recherches en ergonomie}

Depuis les premières analyses en 1946 sur le travail des « facteurs enregistrants » (Pacaud, 1946), les études sur les « relations de service » se sont étendues à de nombreux métiers (Weill-Fassina, \& Caroly, 2003). Cette évolution est à relier au développement du secteur tertiaire, aux difficultés des gestionnaires pour définir et organiser ces tâches et évaluer la qualité de service et aux difficultés rencontrées par le personnel. Ces difficultés sont à l'origine des demandes de recherches ou d'interventions.

La diversification des métiers a permis de distinguer plusieurs types de relation (Gonzalez, WeillFassina, \& Claire-Louisor, 2001), caractérisables en terme de buts, de personnes visées, de contextes organisationnels :

- des conseillers funéraires ayant des relations commerciales avec leur client (Caroly, \& Trompette, 2006) ;

- des guichetiers ayant des relations administratives avec des usagers (Caroly, 2002 ; Caroly, \& Weill - Fassina, 2004a);

- des infirmières en hôpital développant des relations de soins avec leurs patients (Caroly, 2005);

- des auxiliaires de puériculture en crèche ayant des relations psycho-socio-éducatives avec les enfants bénéficiaires (Gonzalez, 2004 ; Gonzalez, \& Weill-Fassina, 2005) ;

- des éducateurs ayant des relations psycho-socio-éducatives avec des enfants et adolescents en difficulté (Claire-Louisor, 2004 ; Claire-Louisor, \& Weill-Fassina, 2005) ;

— des policiers ayant des « relations civiques » de surveillance, prévention, protection, répression avec divers interlocuteurs (Boussard, Loriol, \& Caroly, 2006).

Dans tous les cas, «le service à la personne » est une dimension essentielle de l'activité, son principal «motif». Ce sont des «activités immatérielles et relationnelles... dont les effets ne sont pas tangibles, dans le sens où ils ne sont pas mesurables » (Du Tertre, 2005).

\section{2.-Évolution des perspectives théoriques d'analyse des activités de service}

La place de la personne dans le processus dynamique de l'activité a orienté l'analyse ergonomique de l'activité du " prestataire de service » pour cerner les aspects et les difficultés spécifiques aux différents métiers. Cette évolution des focales d'analyse repose principalement sur trois approches théoriques :

- L'approche behavioriste étudie les effets de variables de situations sur les comportements observables. Pertinente pour analyser les effets des conditions de travail sur l'efficacité et la santé, cette approche se révèle insuffisante pour comprendre les processus mentaux soustendant les comportements. Or ils s'avèrent importants dans les activités de service.

- L'approche interactionniste place au cœur de l'analyse non pas l'opérateur mais le réseau des interactions entre les membres du groupe de travail dont l'organisation émerge de ces interactions.

- L'approche « dialogique » place l'usager comme acteur d'une situation de co-production. Dès 1968, Goffman avait modélisé les interactions entre l'opérateur et son « client » en différenciant les communications techniques, contractuelles et relationnelles. Ce qui implique 
une situation de coproduction et un travail coopératif (Falzon, \& Lapeyrière, 1998).

Cependant, cette « coopération » n'a pas qu'un sens positif et peut présenter des aspects conflictuels. D'après l'analyse de l'activité des douaniers, les tensions avec les usagers lors de contrôles plus ou moins attendus selon les services concernés ont des effets stressants variables sur leur santé (Prunier, 1997).

\section{3.-Un modèle quadripolaire des relations de service}

D'après cette triple approche, les activités de relation de service résultent de compromis entre quatre pôles (Flageul-Caroly, 2001 ; Weill-Fassina, \& Pastré, 2004) :

— un pôle « Système » référant à l'entreprise avec ses buts et ses moyens disponibles (matériel, équipement, règles, procédures, structure hiérarchique);

— un pôle « Soi » référant à l'agent avec ses propres buts, sa subjectivité, sa formation, son expérience, ses possibilités physiologiques et psychologiques, le sens qu'il attribue à son travail ;

— un pôle « autres » référant aux buts, obligations et exigences des autres (collègues du même métier, équipe inter-métiers, réseau de partenaires) ;

— un pôle « personne-à-qui-s'adresse-le-service »; dite selon les cas « client», « patient », « bénéficiaire », « usager », " public », entourage, famille, etc.

Les activités de service sont intégrées dans des « systèmes complexes» (Lemoigne, 1995), caractérisés par des unités en interactions, des processus dynamiques d'interaction; des incertitudes sur l'évolution de la situation, une évaluation problématique du service. L'activité est « située » dans un contexte environnemental et social en évolution.

Pour le prestataire, la compréhension de l'autre, la construction de stratégies à adopter, les compromis élaborés sont essentiels pour atteindre son but.

Pour l'ergonome, ces exigences impliquent, de comprendre les mécanismes affectifs et cognitifs qui orientent et régulent ces comportements en interaction avec le milieu en tenant compte du travail collectif auquel ils participent (Weill-Fassina, \& Benchekroun, 2000). Cependant, les analyses mettent rarement les quatre pôles simultanément en relation. Le modèle multipolaire passe à l'arrière-plan au profit de relations privilégiées selon le type de métier considéré et les demandes d'intervention.

\section{4.-Orientations des analyses des processus sous-tendant les « activités de service aux personnes"}

Les demandes relatant un mal-être des opérateurs (TMS, stress, manque de reconnaissance ...) sont à l'origine des recherches présentées. L'organisation fixe les objectifs du travail mais les descriptions de ces métiers insistent plus sur leurs aspects matériels visibles que sur ce qui fonde la relation du prestataire avec l'usager, les moyens pour parvenir au but, les règles et procédures, ce qui laisse aux opérateurs des marges de manœuvre potentielles.

Les analyses ergonomiques ont eu pour but de dégager les propriétés essentielles des activités sousjacentes aux comportements observés pour établir un diagnostic de la situation et répondre à la demande. Dans des perspectives psychologiques et/ou organisationnelles, l'hypothèse de compromis entre les exigences des différents pôles a conduit à s'interroger sur les représentations et les stratégies des opérateurs, le développement de leurs compétences dans les processus de travail collectif auxquels ils participent. Ces études montrent que les activités individuelles ou collectives consistent à « élaborer » ou « réélaborer» des diagnostics, des plans d'action, des règles pour adapter leurs actions aux circonstances et assurer la qualité de service attendue. Nous reprendrons quelques-unes de nos recherches pour l'illustrer. 


\section{2.- Relation soi-bénéficiaire / système-autrui : élaboration d'une représentation de l'évolution de l'enfant en foyer pour construire un projet socio-éducatif}

La mission des éducateurs est d'accueillir, d'observer et d'orienter des mineurs en difficulté. Ils prennent en charge l'enfant : subviennent à ses besoins et élaborent avec lui un projet qui constitue le principal motif de leur activité. Cette élaboration se fait par un travail d'équipe (collègues, instances judiciaires, assistante sociale, enseignants, médecin, famille) fondé sur l'observation de l'enfant.

Historiquement, «l'analyse de l'activité » a porté sur l'effet des conditions de travail sur le comportement des éducateurs : par exemple, poids des décisions politiques sur l'adoption de stratégies coûteuses pour les auxiliaires familiales (Cloutier, David, Teiger, \& Prévost, 1999), impact de la durée de l'entretien sur le recueil de renseignements socio-educatifs destiné au juge au détriment de sollicitations sur le projet de l'enfant (Villatte, 2000), effet de l'environnement social sur le stress d'éducateurs de rue (Messenti, \& Kurth, 2004). D'autres recherches portent sur les manières de se comporter auprès de l'enfant : par exemple, observation d'une distance dans la relation avec les enfants selon l'âge des éducateurs (Caroly, 1997)

Cependant interagir avec le bénéficiaire implique de pouvoir se fonder sur une « représentation opérative » de sa situation pour intervenir (Weill-Fassina, Rabardel, \& Dubois, 1993).

\section{1.-« Comprendre le bénéficiaire », un point clef dans le travail des éducateurs}

Des notes quotidiennes sont prises pour « comprendre » l'enfant, le suivre et déterminer l'organisation de sa prise en charge.

Les observations et leurs notations font l'objet de prescriptions floues et s'apparentent à une des discussions collectives. L'analyse de la relation de l'éducateur avec l'enfant est donc l'objet de recherche pour comprendre la construction d'une représentation de l'évolution de l'enfant par l'éducateur.

\section{2.-Activités des éducateurs mis en évidence par diverses techniques d'analyse}

L'observation est par définition inobservable par définition ; celle d'un enfant se prolonge dans un temps non déterminé, se répartit entre plusieurs éducateurs et se distribue, en situation, sur un groupe d'enfants. Les notations quotidiennes dans les cahiers de transmission sont des transcriptions des observations de l'éducateur, de ses réflexions et résultent d'un processus d'objectivation. Ce sont donc des « traces de l'activité » dont l'analyse permet d'inférer les interactions entre l'éducateur et l'enfant.

L'analyse de contenu des notations de 13 dossiers d'enfants (Claire-Louisor, 2004) a permis de catégoriser les thèmes et les objets d'observation retenus par les éducateurs. Ces informations sont constitutives de leurs représentations mais, étant décontextualisées, elles ne sont pas interprétables par l'analyste.

L'auto confrontation vise à susciter une réflexion a posteriori sur ces observations et une prise de conscience concernant leur sens pour « comprendre» l'histoire de l'enfant. Les protocoles verbaux recueillis montrent que cette activité réflexive conduit à des réinterprétations, des découvertes, de nouveaux questionnements qui laissent apparaître à la fois la représentation construite dans l'action, ses difficultés d'élaboration, la construction d'une nouvelle représentation de l'histoire de l'enfant au foyer et une meilleure compréhension des obstacles rencontrés dans l'élaboration du projet.

La comparaison de plusieurs histoires d'enfants a permis de dégager un modèle en quatre phases du processus d'adaptation des enfants en foyer (découverte, installation, stabilisation, attente) avec des critères d'arrêt marqués par des changements de comportements et des critères d'alerte liés à la durée de certaines phases. L'absence de repérage de phases témoignent d'un manque de congruence de buts 
entre un " projet visée » de l'enfant qui n'a pas été identifié et le projet programmatique des éducateurs. Cette élaboration d'un modèle du processus d'adaptation des enfants donne une référence à la prise en charge socio-éducative en situation.

\section{3.- Relations soi - autrui / patient - système : Elaboration collective de la planification d'une activité par le personnel soignant}

Dans une première période de nombreuses études ont porté sur les effets des conditions de travail des infirmières (horaires, charge, pression temporelle, tâches interférentes...) sur leur santé et leurs rapports au patient. A l'hôpital, plusieurs professionnels travaillent dans le même espace, avec des tâches plus ou moins spécifiques auprès des patients. L'objet produit dans la relation de service est issu de l'interaction entre l'agent et le patient qui s'élabore dans le système de travail (environnement, outils, moyens, objectifs) (Falzon, 1997). Les études se sont déplacées des interactions avec le patient à l'organisation du travail collectif. L'interaction se partage entre les membres d'une équipe (Borzeix, 1992 ; Dartevelle, 1992 ; Grosjean, \& Lacoste, 2002). Ceci implique un agencement organisationnel collectif fondé sur une dynamique de frontières entre divers processus de travail.

L'analyse des relations entre soi-autrui par rapport à système-patient vise à comprendre comment l'organisation d'activités collectives peut être source de dysfonctionnement et de dégradation de la qualité de service et à répondre aux problèmes de répartition et de planification du service au patient dans une équipe de professionnels (infirmières, aides, soignants, agents techniques).

\section{1.-« Différentes formes de travail collectif », un point clef dans l'activité des soignants}

Le terme de coopération habituellement utilisé n'est pas synonyme de travail collectif (De la Garza, \& Weill-Fassina, 2000). Les activités collectives prennent différentes formes selon les buts des opérateurs et leurs coordinations dans le temps et l'espace. Le travail collectif du personnel infirmier illustre ces différentes formes :

- La co-action concerne des situations où les buts sont différents pour les opérateurs, mais intégrés dans une activité commune à long terme (Savoyant, 1977). C'est le cas de soignant lorsque l'un s'occupe du pansement et l'autre de la toilette pour préparer le patient à sortir.

- La collaboration correspond à une situation où les buts à court terme sont identiques avec des actions différentes pour chaque opérateur (Rogalski, 1994). Pour installer le patient dans son lit pour manger, un soignant rehausse le lit et l'autre rapproche la table.

- La coopération caractérise une activité collective dans laquelle les opérateurs travaillent ensemble sur le même objet visant au même but proximal (De la Garza, 1995). L'infirmière et l'aide-soignante refont ensemble le lit d'une personne dépendante.

- L'aide ou l'entraide correspond à une situation où un professionnel vient aider un autre, sans que ce dernier le lui ait demandé (Assunçao, 1998). Une aide-soignante voit les difficultés d'une collègue pour déplacer un chariot dans un couloir encombré et vient l'aider, souvent à charge de revanche.

Ces différentes formes d'activités collectives marquent la diversité des relations entre l'agent et ses collègues. Observer la façon dont les professionnels se coordonnent, permet de rendre compte de la qualité du service au patient.

\section{2.-La coordination des activités des soignants autour du patient : techniques d'analyse et transformation du travail}

La méthodologie repose sur l'identification des dysfonctionnements et de leurs causes, et sur une 
discussion de groupe entre professionnels leur permettant de se concerter et de se coordonner. Cette activité méta-fonctionnelle vise une réélaboration de la planification de leur travail autour d'un référentiel commun.

Les résultats sont les suivants :

- Les troubles de santé (usure, fatigue, TMS, etc.) et les difficultés à assurer la qualité de service au patient sont les principaux dysfonctionnements liés au manque d'organisation collective $\mathrm{du}$ travail.

- Une des conditions de la coordination repose sur la durée de concertation préalable à l'activité dans des espaces-temps hors situation d'interaction avec le patient (transmission ou relève).

- La planification des tâches suppose que la coordination inter-métiers soit un mode de fonctionnement effectif de l'équipe. Ainsi, servir le petit déjeûner nécessite une coordination des infirmières, des aides-soignantes et des agents de services hospitaliers. La concertation porte sur les possibilités d'agir à leur discrétion dans le cadre d'une organisation prescrite floue.

- Les modes opératoires mis en œuvre individuellement en référence à un collectif de travail permettent des compensations des perturbations liées aux conditions externes des tâches et à leur environnement.

- Le collectif de travail n'est pas une donnée. C'est une construction à partir d'un travail collectif. La simulation de séquences de travail coordonnées et les auto-confrontations de groupe favorisent la connaissance du travail de l'autre, l'émergence de références communes sur l'action collective et la conception d'une organisation collective du travail.

\section{4.- Relations soi-système-interlocuteur / autrui. Des réélaborations de règles dans l'action par les policiers pour tenir compte des risques encourus}

Les policiers ont pour mission de faire respecter l'ordre, d'assurer la sécurité et de lutter contre la délinquance. La définition de leur tâche comprend des procédures strictes sur la manière d'intervenir auprès des agresseurs et de règles administratives relatives à l'application de la Loi.

Depuis 1996, les relations de services ont été étudiées par rapport à la « transgression des règles » et à leurs différents modes d'application (Girin, \& Grosjean, 1996). L'analyse d'un rapport aux règles est fondée sur l'étude des relations contractuelles entre l'agent, l'interlocuteur et l'organisation. La mise en évidence de normes de groupe, de stratégies de contournement des règles, de ruses du client dans l'utilisation des règles, correspond à une nouvelle façon d'explorer les activités de service (Montjardet, 1996; Caroly, \& Scheller, 1999). Explorer les relations entre soi, l'interlocuteur et le système tout en tenant compte de l'équipe, permet de resituer ces stratégies dans la dynamique de «la régulation du processus de travail », en référence à la théorie de «l'Agir Organisationnel » (Maggi, 2003).

Chez les policiers, les relations entre soi, le système, l'agresseur et/ou la victime ont été analysées en rapport avec l'équipe et les partenaires (juge, pompiers, médecin,...). L'analyse ergonomique vise à comprendre les modalités de "régulation contextuelle à l'action » c'est-à-dire les modalités de réélaborations de règles dans la gestion de situations potentiellement conflictuelles entre policiers et interlocuteurs selon le contexte de l'interaction.

\section{1.-Les formes de re-élaboration de règles, un point clef de l'activité des policiers}

Un suivi des activités des policiers sur le terrain et des entretiens consécutifs ont montré qu'au quo- 
tidien, les policiers «ajustent des règles » selon la population interpellée, le contexte social et les circonstances. Considérer l'écart à la règle formelle comme due à une formation insuffisante, une erreur individuelle, un écart à la norme n'est pas envisageable si l'on conçoit la modification des règles comme un mode de régulation de perturbations provenant de l'interlocuteur et comme un mode collectif de gestion de situations à risques.

Les formes de re-élaborations des règles varient selon la conception personnelle de l'agent, son rapport à l'interlocuteur et à ses collègues :

- Non-respect de la Loi : si le policier enfreint la Loi, ses collègues le jugent négativement et ne le soutiennent pas. Par exemple, dans une course poursuite, pour rattraper un délinquant, dépasser une vitesse autorisée peut accentuer le risque que la personne poursuivie accélère aussi et provoque ou ait un accident. Pour la hiérarchie ce non-respect serait contrevenir au code de la route.

- Non-application de la règle : dans la pratique, il arrive que les policiers n'appliquent pas les règles administratives dans certaines situations difficiles d'intervention. Cette non-application, si elle est courante et commune à l'équipe, peut devenir une « règle de contrôle ». Par exemple, contrairement aux règles officielles, des policiers organisent des livraisons contrôlées de drogue pour infiltrer des réseaux (Montjardet, 1996).

- Contournement des règles : pour s'accorder avec le milieu, le contexte familial et social de la victime et/ou de l'agresseur, le policier est amené à « tricher» avec les règles pour assurer la prévention. Par exemple, lors d'un contrôle, il peut ne pas insister sur l'absence de papiers d'identité de personnes en situation illégale sachant que l'interlocuteur possède une arme à feu et que la situation risque de dégénérer.

- Réĺlaborations collectives des règles : dans certaines situations de règles contradictoires ou de conflits avec l'interlocuteur, les policiers réélaborent collectivement de nouvelles règles pour trouver un accord avec leurs interlocuteurs. Par exemple, lorsqu'un « jeune » circule en scooter sans casque, le policier est confronté à un conflit entre la règle qui prévoit de l'arrêter et le risque d'une fuite, qui pourrait provoquer un accident du « jeune » dont il serait responsable. La règle réélaborée et admise par l'équipe consiste à ne pas arrêter un « jeune » sans casque, règle connue des contrevenants.

Ainsi, les policiers « reélaborent» les règles d'action pour les adapter au contexte du terrain. Ces réélaborations résultent de compromis entre les règles préalables de l'organisation et les risques encourus en temps réel pour prévenir l'aggravation de la situation. La plupart de ces régulations dénotent des conduites autonomes de la part des policiers par rapport aux règles préalables. Elles s'inscrivent dans le cadre de négociations avec le collectif tout en tenant compte des astreintes de la situation de terrain et en particulier des risques pour l'interlocuteur, l'environnement, les collègues et soi-même.

\section{2.-Facteurs individuels et collectifs intervenant dans les re-élaborations de règles}

L'analyse souligne l'influence des difficultés d'application des règles et des consignes données de l'encadrement, du rapport à l'équipe, des contraintes environnementales, du comportement de l'interlocuteur et des objectifs de l'agent sur les différentes formes de réélaborations des règles :

- la conception personnelle du travail joue un rôle dans la re-élaboration des règles selon la population. La dimension émotionnelle peut se traduire par des contournements ;

- il existe des différences entre générations. Les conflits de règles sont plus exacerbés chez les âgés expérimentés qui ont connu l'ancien système de règles que chez les jeunes nouvellement formés ;

- l'intégration des règles évolue au fil du temps. Les anciens sont davantage en capacité de 
discuter avec le collectif (y compris encadrement) de la fragilité de certaines règles et de la nécessité de les reconstruire ;

- le nombre de non -applications des règles est doublé lorsque l'équipe est stable (faible turnover) et que les patrouilles sont fixes (continuité des policiers dans les voitures, etc). Ces nonapplications sont significatives de régulations de situations à risque ;

- les re-élaborations de règles dépendent en partie de la collaboration entre les services (par exemple, relations entre back office / front office ; relations entre police de proximité / brigade anti-criminelle, etc.) ;

- l'interlocuteur cherche de son côté à transiger avec les règles en évoquant sa connaissance de la loi et en négociant l'application des règles qu'il connaît bien.

\section{5.- Les relations soi-client-système-autrui. Des réélaborations de règles sur le long terme comme symptôme d'évolution des compétences des guichetiers}

Les tâches des guichetiers de La Poste sont multiples (vendre des produits, accueillir du public en précarité, conseiller le client et gérer la file d'attente, etc.) et multidimensionnelles : dimension commerciale avec la vente de produits financiers et postaux ; contractuelle reposant sur l'utilisation de règles administratives dans la transaction ; relationnelle basée sur des scripts de comportement pour bien accueillir les usagers.

Depuis 1999, les recherches sur les relations de services montrent l'existence de conflits de buts et de moyens dans les interactions avec l'usager par rapport aux règles du système (Cloutier, David, Teiger, \& Prévost, 1999 ; Flageul-Caroly, 2001). Ces conflits sont sources de situations critiques moins par rapport à l'usager que par rapport aux choix et valeurs de l'opérateur.

Pour les guichetiers, les relations entre pôles de l'activité sont sources de situations critiques. Les régulations mises en œuvre pour résoudre ces conflits reposent sur des recherches d'équilibre et la construction de compromis entre soi-client-système-autrui. L'analyse de leurs activités de service est focalisée sur l'évolution des stratégies individuelles pour gérer ces conflits de buts et de moyens entre les quatre pôles de l'activité, et sur le développement de leurs compétences avec l'âge et l'ancienneté selon qu'il participe ou non à un collectif de travail.

\section{1.-Evolutions des régulations des situations critiques}

La méthode d'analyse de l'activité pour rendre compte de l'évolution des compétences, dépasse la description des stratégies pour inférer l'évolution de l'organisation de l'action avec l'expérience (Weill-Fassina, \& Pastré, 2004). D'où une comparaison entre novices et anciens.

Le développement des compétences se caractérise par une recherche d'équilibre entre les obligations du système et leur conception personnelle de la qualité de service (Caroly, \& Weill-Fassina, 2004b) et se manifeste par :

- l'extension des champs pris en compte dans l'action avec l'expérience. Ainsi, les anciens vendent selon le besoin du client en l'intégrant dans une réélaboration des règles.

- l'augmentation du champ temporel pris en considération, corollaire de l'extension des champs. Avec l'expérience, le champ temporel, d'abord très réduit permet des anticipations et donc des régulations pré-correctives pour compenser des aléas devenus prévisibles. D'où des réorganisations de séquences d'action. Ainsi, pour les déplacements fréquents en back office pour se réapprovisionner en argent et objets de valeurs pouvant être source de conflit avec les usagers et faire obstacle à la vente, les anciens combinent un déplacement obligatoire à la caisse pour se réapprovisionner. 
- les réélaborations de règles. Avec l'expérience, les guichetiers qui, au début, tendent à appliquer les règles prescrites, les intègrent au reste de leur activité. Ils les réélaborent selon les besoins du client, de leur connaissance des autres contraintes du système. Ainsi, devant l'impossibilité de vendre des paquets d'enveloppes pré-timbrées, ils les vendent progressivement en profitant de diverses occasions.

- ces évolutions à long terme ne sont possibles que dans le cadre d'une dynamique collective de réorganisation du travail (Maggi, 2003 ; Caroly, \& Clot, 2004).

\section{2.-L'activité collective comme support à la réélaboration des stratégies multifonctionnelles des guichetiers}

Faciliter les modalités de développement des compétences dépend de l'élaboration des règles de métiers (Cru, 1995 ; Clot, 1999). D'où la comparaison du fonctionnement des équipes de travail de plusieurs bureaux de Poste concernant la mise en œuvre de régulations collectives, l'élaboration de compromis entre des logiques de santé, efficacité, qualité, etc.

Les résultats de cette recherche montrent :

- l'existence de conditions à l'élaboration de stratégies multifonctionnelles : leur reconnaissance par les pairs est nécessaire pour combiner plusieurs objectifs (par exemple qualité de service, préservation de sa santé, gestion de la file d'attente pour le guichetier);

- le développement d'activités méta-fonctionnelles dépend de pratiques réflexives spontanées et partagées (petits carnets, façon de compter les billets pour ne pas faire d'erreur, etc.) et de pratiques collectives (cahier d'ouverture de compte, outils formels et informels de consignes administratives ou comptables, ...);

- la résolution des conflits de buts évolue au fil de l'âge et de l'expérience vers une organisation de l'action plus étendue, à condition que le travail collectif facilite la création de marges de manœuvre en terme d'autonomie et de discrétion ;

- le collectif de travail favorise le développement des compétences individuelles si son élaboration se fonde sur la transmission des connaissances, le partage des savoir-faire et une confiance réciproque ;

- les possibilités de développement des styles individuels favorisent la mise en oeuvre de stratégies de prudence ou d'économie pour préserver sa santé.

\section{6.- Conclusion sur les théories d'analyse de l'activité}

1- Il ressort de ces quatre recherches que, dans les métiers de service aux personnes, l'élaboration et/ou la re-élaboration des représentations des situations, des plans d'action, des règles, constitue une part essentielle des activités individuelles et collectives des " prestataires de service " pour adapter leurs actions aux circonstances et assurer la qualité de service attendue.

Ces réélaborations s'inscrivent d'un point de vue organisationnel dans le cadre de la régulation du processus de travail (Maggi, 2003). Elles sont considérées ici d'un point de vue ergonomique et psychologique pour comprendre pourquoi et comment les opérateurs les mettent en place dans le déroulement de leurs activités.

2- En ce qui concerne les caractéristiques des activités individuelles, les analyses reposent sur deux cadres théoriques :

Les recherches ont essayé d'intégrer différentes composantes de l'activité, en référence à la théorie développée par Leontiev : activité, actions, opérations ;

Les analyses des activités métafonctionnelles, issues des entretiens consécutifs et d'auto-confronta- 
tion des agents, ont permis d'articuler trois niveaux d'activités, en référence au développement de la structuration de la conceptualisation décrit par Piaget (1975):

- le niveau de l'action proprement dit qui fait l'objet d'observation des comportements ;

- le niveau réflexif sur les propriétés des objets, des interlocuteurs et du contexte qui fondent les représentations des situations et leurs évolutions ;

- le niveau réfléchissant sur les propriétés et conséquences des règles, des procédures et des actions et qui semble central dans les processus de re-élaborations.

Ces différents niveaux des activités individuelles se manifestent dans l'organisation de l'action qui est marquée, selon les cas, par le passage de l'utilisation directe d'éléments concrets de la situation à des transformations des représentations, des règles..., à la création de marges de manœuvre ou à l'augmentation du nombre des dimensions prises en compte dans l'action.

Les deux dernières recherches ont fait apparaître des évolutions de cette organisation de l'action tout au long de la vie professionnelle. Par contre, dans les deux premières recherches, ces évolutions semblent être une conséquence directe de méthodes d'analyse qui ont demandé aux opérateurs une nouvelle réflexion sur leurs actions et leur but.

3- Par ailleurs, le modèle quadripolaire qui sous-tend les recherches citées et les résultats obtenus montre clairement l'impossibilité de considérer ces activités en dehors du contexte du travail collectif auquel elles participent. Plusieurs caractéristiques des activités collectives ont pu être soulignées lors de l'analyse des différentes activités de service :

- leurs différentes formes allant de la co-action à la coopération ;

- leurs différentes organisations allant du groupe peu structuré, à l'équipe structurée formellement, au collectif de travail ;

- leur possibilité de construction et d'évolution.

4- Par rapport aux débats actuels (Clot, 1999 ; Maggi, \& Lagrange, 2002) sur les relations qui existent entre activités individuelles et collectives, les analyses des activités de service nous permettent de distinguer :

- les cas des éducateurs et des infirmières où le travail collectif émerge des interactions des différents acteurs devant la nécessité de résoudre un problème ;

- les cas des policiers et des guichetiers où le travail individuel émerge d'un travail collectif ou d'un collectif de travail ;

Selon ces différents exemples, il serait actuellement hasardeux de définir de manière univoque les rapports entre activités de service individuelles et collectives. Il semble que les caractéristiques mises en évidence dépendent autant du moment et du lieu de l'observation que du problème posé tant à l'opérateur qu'à l'observateur.

5- Suscitées par des demandes faisant état de difficultés dans la réalisation des activités de service, les recherches citées ont cependant toutes insisté sur les possibilités et les modalités de régulation des situations par les « prestataires de service », et donc sur les aspects positifs de " l'adaptation aux contraintes de situation ou du milieu ». Cependant, tous les opérateurs ne trouvent pas la voie pour compenser les perturbations ressenties. Pour ceux-ci, les conditions de travail peuvent être sources d'handicaps de situation, de souffrances, de burn-out, voire de suicide, d'erreurs, d'incidents ou d'accidents. Il serait nécessaire de mieux comprendre les sources de ces différences.

6- Ainsi selon les recherches citées, il a été nécessaire de faire appel à différentes théories de l'activité et théories de l'action pour rendre compte de la diversité des aspects de l'activité qui interviennent dans la construction et la re-élaboration des représentations, des règles et des relations avec autrui. Afin de comprendre les diverses modalités de réélaboration des règles par les opérateurs et leur encadrement, il semble nécessaire de faire évoluer l'orientation de l'analyse de l'activité vers une approche multidisciplinaire qui reposerait sur une ouverture de l'ergonomie aux théories développe- 
mentales, de l'organisation et du management et qui chercherait à coordonner des connaissances en psychologie, en sociologie et en gestion.

\section{RÉFÉRENCEMENT}

Caroly, S. \& Weill-Fassina, A. (2007). En quoi différentes approches de l'activité collective des relations de services interrogent la pluralité des modèles de l'activité en ergonomie ?@ctivités, 4 (1),pp. 85-98, http://www.activites.org/v4n1/v4n1.pdf

\section{RÉFÉRENCES}

Avila Assunção, A. (1998). De la déficience à la gestion collective du travail : les troubles musculosquelettiques dans la restauration collective. Thèse de doctorat d'ergonomie, Paris V: Ecole pratique des hautes Etudes.

Borzeix, A. (1992). L'évitement et l'engagement refusé. In A. Borzeix, \& B. Gardin, (Eds.), Langage et activités de service, Cahier Langage et Travail, n 4, 1-7.

Boussard, V., Loriol, M., \& Caroly, S. (2006).Catégorisation des usagers et rhétorique professionnelle : le cas des policiers sur la voie publique. Sociologie du travail, 48 (2), 137-278.

Caroly, S. (1997). Vieillissement et expérience : analyse de l'activité des éducateurs en foyer. Mémoire de DEA d'ergonomie. LEPC-CNAM-Université Toulouse Le Mirail.

Caroly, S. (2002). Différences de gestion collective des situations critiques dans les activités de service selon deux types d'organisation du travail. PISTES, 4 (1), http://www.pistes.uqam.ca

Caroly, S. (2005). Conditions de la prévention durable des lombalgies des personnels hospitaliers : intervenir sur l'organisation collective du travail. $40^{\text {ème }}$ Congrès de la SELF, Ergonomie et Prévention durable, 2123 septembre 2005 (pp. 266-274). L'Ile de la Réunion.

Caroly, S, \& Clot, Y. (2004). Du travail collectif au collectif de travail. Des conditions de développement des stratégies d'expérience. Comparaison de deux bureaux. Formation et Emploi, nº88, 43-55.

Caroly, S, \& Scheller, L. (1999). Expérience et compétences des guichetiers de la Poste dans leurs rapports à la règle. In Actes du XXXIVè Congrès de la SELF, Ergonomie et relation santéltravail (pp. 221-229). Caen,

Caroly, S., \& Trompette, P. (2006). De la compétence de service aux compétences de coordination et d'orchestration : Autour du conseiller funéraire. PISTES, 1 (1), http://www.pistes.uqam.ca

Caroly, S, \& Weill-Fassina, A. (2004a). Evolutions des régulations de situations critiques au cours de la vie professionnelle dans des activités de relations de service. Le Travail Humain. 67 (4), 304-327.

Caroly, S., \& Weill-Fassina, A. (2004b). Développement des compétences professionnelles et collectif de travail aux guichets de la Poste. Bulletin de psychologie. Tome 57 (1)/469/ janvier-février 2004, 49-53.

Claire-Louisor, J. (2004). L'observation professionnelle en situation de prise en charge socio-éducative. Thèse de Doctorat en ergonomie. Laboratoire d'ergonomie physiologique et cognitive. Ecole pratique des hautes études. Paris.

Claire-Louisor, J , \& Weill-Fassina, A. (2005). Modalités d'adaptation d'enfants en situation de prise en charge socio-éducative. In E. Callu, J.P. Jurmond, \& A. Vambeau (Eds), La place des jeunes dans la cité. Tome 2. Espaces de rue, espaces de paroles. Paris: L'Harmattan.

Clot, Y. (1999). La fonction psychologique du travail. Paris: PUF.

Cloutier, E., David, H., Teiger, C., \& Prevost, J. (1999). Les compétences des auxiliaires familiales et sociales expérimentées dans la gestion des contraintes de temps et des risques à leur santé. Formation Emploi (numéro spécial), ${ }^{\circ} 67,63-75$.

Cru, D. (1995). Règles de métier. Langue de métier. Diplôme d’Ergonomie. Paris: Ecole Pratique des Hautes Etudes. 
Dartevelle, M. (1992). Langage et maintien de l'ordre. In A. Borzeix, B. Gardin (Eds.). Langage et activités de service. Cahier Langage et travail, $\mathrm{n}^{\circ} 4,9-24$.

De la Garza, C. (1995). Gestion individuelles et collectives du danger et du risque dans la maintenance d'infrastructures ferroviaires. Thèse de doctorat d'Ergonomie. Paris V: LEPC-EPHE.

De la Garza, C., \& Weill-Fassina, A. (2000). Régulations horizontales et verticales du risque. In A. WeillFassina, A., \& T.H. Benchekroun, Le travail collectif : perspectives actuelles en ergonomie (pp. 217-234). Toulouse: Octarès Éditions.

Du Tertre, C. (2005). Services immatériels et relationnels : intensité du travail et santé. @ctivités, 2 (1), http://www.activites.org

Falzon, P. (1997). Les relations de service. Performances, n89, 6-7

Falzon, P, \& Lapeyrière, S. (1998). L'usager et l'opérateur : ergonomie et relations de service. Le Travail Humain, 61, 69-90.

Flageul-Caroly, S. (2001). Régulations individuelles et collectives des situations critiques dans un secteur des services : le guichet de la Poste. Paris V: Thèse d'ergonomie EPHE- LEPC.

Girin, J, \& Grosjean, M. (1996). La transgression des règles au travail. Paris: l’Harmattan.

Gonzalez, R. (2004). Du macro au micro : les régulations des relations de service en crèches. Paris: Thèse de Doctorat en Ergonomie. Laboratoire d'Ergonomie du CNAM.

Gonzalez, R., Claire-Louisor, J., \& Weill-Fassina, A. (2001). Les activités d'interventions psycho-socioéducatives : une catégorie spécifique de relation de service. Congrès de la SELF-ACE, Montréal, Canada.

Gonzalez, R, \& Weill-Fassina, A. (2005). Modalités de régulation du processus de travail dans les activités de service en crèche. @ctivités. 2 (2), http://www.activites.org

Grosjean, M., \& Lacoste, M. (1999). Communication et intelligence collective : le travail à l'hôpital. Paris: PUF.

Lemoigne, J.L. (1995). La modélisation des systèmes complexes. Paris: Dunod.

Maggi, B. (2003). De l'agir organisationnel. Un point de vue sur le travail, le bien-être, l'apprentissage. Toulouse: Octarès Éditions.

Maggi, B., \& Lagrange, V. (2002). Le travail collectif dans l'industrie à risque. Six points de vue de chercheurs étayés et discutés. Toulouse: Octarès Éditions.

Messenti, M., \& Kurth, S. (2004). Recherches et pratiques des normes ergonomiques. In P. Rey, E. Ollagnier, V. Gonik, \& D. Ramaciotti (Eds.), Actes du XXXIXe Congrès SELF, Ergonomie et normalisation (pp. 313318). Genève, Suisse.

Montjardet, D. (1996). Règles, procédures et transgressions dans le travail policier. In J. Girin, \& M. Grosjean (Eds.), La transgression des règles au travail (pp. 83-94). Paris: l'Harmattan.

Pacaud, S. (1946). Recherches sur la sélection psychotechnique des agents de gare, dits « facteurs enregistrants ». Le Travail Humain, 9, 23-73.

Piaget, J. (1975). L’équilibration des structures cognitives, problème central du développement. Études d'épistémologie génétique, vol. 13. Paris: PUF.

Prunier, S. (1997). Contraintes des horaires et exigences des tâches : la double détermination des effets du travail posté. Santé et vie socio-familiale des agents des Douanes. L'Ecole Pratique des Hautes Etudes, Paris: Thèse de Doctorat en Ergonomie.

Rogalski, J. (1994). Formation aux activités collectives. Le Travail humain, 57 (4), 367-386.

Savoyant, A. (1977). Coordination et communication dans une équipe de travail. Le Travail Humain, 40 (1), 41-54

Villatte, R. (2000). Approche ergonomique des pratiques de reccueil de resnseignements socio-éducatifs (RRSE) des éducateurs de la protection judiciaire de la jeunesse. In B. Mélier, \& Y. Quéinnec (Eds.), Communication et travail. Actes du XXXVe Congrès de la SELF (pp. 570-584). Toulouse: Octarès Éditions. 
Weill-Fassina, A, \& Benchekroun, T. (2000). Le travail collectif : perspectives actuelles en ergonomie. Toulouse: Octarès Éditions.

Weill-Fassina, A, \& Caroly, S. (2003) Evolution des recherches ergonomiques sur les activités de service. Actes des Journées de Bordeaux sur la pratique de l'ergonomie (pp. 105-114). Université Bordeaux. ISPED: LESC-ARACT.

Weill-Fassina, A., \& Pastré, P. (2004). Les compétences professionnelles et leur développement. In P. Falzon (Ed.), Ergonomie (pp. 213-232). Paris: PUF.

Weill-Fassina, A., Rabardel, P., \& Dubois, D. (Eds.). (1993). Représentations pour l'action. Toulouse: Octarès Éditions.

\section{RÉSUMÉ}

L'objectif de cet article est de montrer que la diversité des activités de relations de service selon leurs buts et les demandes d'intervention nécessite d'être prudent sur ce que l'on nomme «activité ». L'étude des tâches de différents métiers des services (soignant, guichetier, policier, conseiller funéraire, auxiliaire petite enfance, éducateurs spécialisés, ...) montre qu'ils ne font pas le même travail : leurs objectifs, le type d'interaction entre le «client » et l'agent, les conditions de travail et son organisation, correspondent à divers possibilités de relations de service. Dans un but de transformation de ces situations de travail pour améliorer la santé des agents et l'efficacité du système, il est nécessaire de dépasser une analyse behavioriste, basée sur la description des tâches ou l'observation des comportements pour accéder aux conditions de réalisation du travail. Pour cela, nous avons besoin de mieux prendre en compte des modèles internes de l'activité pour comprendre le fonctionnement physiologique, affectif et cognitif de l'agent. L'activité sera décrite par les régulations mises en œuvre par le travailleur en fonction des ses motivations, de ses buts et de ses possibilités d'interaction avec les conditions environnementales. Le cadre théorique mobilisé est celui de l'analyse pragmatique et systémique de l'activité, qui permet l'existence de différentes théories de l'activité, selon les objectifs de la recherche et de ces conditions.

\section{Mots Clé}

Théorie de l'activité, Relations de services, Régulations, Travail Collectif, Organisation.

\section{RESUMEN}

El objetivo de este artículo es mostrar que la diversidad de las actividades de relaciones de servicio, según sus objetivos y pedidos de intervención, requiere ser prudentes acerca de aquello que se da por llamar « actividad ». El estudio de las tareas de diferentes oficios de servicio (auxiliares de enfermería, agentes de atención al publico, policías, consejeros de funerarias, auxiliares de guardería, educadores especializados,...) muestra que éstos no realizan el mismo trabajo: sus objetivos, el tipo de interacción entre el «cliente » y el agente, las condiciones de trabajo y su organización, corresponden a diferentes posibilidades de relaciones de servicio. Con el objetivo de transformar estas situaciones de trabajo a fines de mejorar la salud de los agentes y la eficacia del sistema, es necesario sobrepasar el análisis conductista, basado en la descripción de las tareas o en la observación de los comportamientos, para acceder a las condiciones de realización del trabajo. Para esto, necesitamos tomar en cuenta en forma más profunda los modelos internos de la actividad a fin de comprender el funcionamiento fisiológico, afectivo y cognitivo del agente. La actividad será descripta mediante las regulaciones desplegadas por el trabajador en función de sus motivaciones, sus objetivos y sus posibilidades de interacción con las condiciones del medio ambiente. El marco teórico retenido es el del análisis pragmático y sistémico de la actividad el que, de acuerdo a los 
objetivos de la investigación y de sus condiciones, permite la existencia de diferentes teorías de la actividad.

Palabras clave

Teoría de la actividad, Relación de servicios, Regulaciones, Trabajo colectivo, Organización. 